\title{
Impaction of a continuous glucose monitoring sensor
}

\author{
Kyong Chan Park ${ }^{1}$, Hwan Jun Choi ${ }^{2}$ \\ ${ }^{1}$ Department of Plastic and Reconstructive Surgery, Soonchunhyang University Bucheon Hospital, Soonchunhyang University College of \\ Medicine, Bucheon; ${ }^{2}$ Department of Plastic and Reconstructive Surgery, Soonchunhyang University Cheonan Hospital, Soonchunhyang \\ University College of Medicine, Cheonan, Korea
}

\begin{abstract}
A 33-year-old man presented to the plastic surgery department for foreign body removal 1 month after the insertion of a continuous glucose monitoring (CGM) sensor (Dexcom G5) in the left upper arm. The patient had used the CGM system for 5 years, and the insertion was done in the usual manner. The entire sensor wire was visible on simple radiography and ultrasonography. In the operating room, and the sensor wire was identified in the intermuscular septum and removed. No foreign body reaction or inflammatory signs were found around the CGM, and the extracted wire measured $2.5 \mathrm{~cm}$. Thus, it was assumed that the whole sensor wire was detached from the transmitter, not fractured. No remnant foreign body was observed on follow-up simple radiography.
\end{abstract}

Keywords Biosensor / Home blood glucose monitoring / Foreign bodies

\author{
Correspondence: Hwan Jun Choi \\ Department of Plastic and \\ Reconstructive Surgery, \\ Soonchunhyang University Cheonan \\ Hospital, Soonchunhyang University \\ College of Medicine, 31 \\ Suncheonhyang 6-gil, Dongnam-gu, \\ Cheonan 31151, Korea \\ Tel: +82-41-570-2195 \\ Fax: +82-41-574-6133 \\ E-mail: medi619@hanmail.net
}

This work was supported by the Soonchunhyang University Research Fund.

Received: January 20, 2021 - Revised: April 14, 2021 • Accepted: May 4, 2021

pISSN: 2234-6163 • elSSN: 2234-6171 • https://doi.org/10.5999/aps.2021.00178• Arch Plast Surg 2021;48:392-394

\section{INTRODUCTION}

The number of diabetes patients is rapidly increasing worldwide; it rose from 108 million in 1980 to 422 million in 2014, and is projected to exceed 500 million cases in 2030 [1]. Traditional measures of glucose control such as hemoglobin Alc or self-monitored blood glucose have limitations regarding the need for daily changes in therapies. Continuous glucose monitoring (CGM) has become increasingly reliable and its efficacy has been demonstrated [2]. Complications of CGM devices have been reported, such as foreign body reaction [3]. Herein, we report our experience with a case of impaction of a CGM sensor wire detached from the device.

\section{CASE}

A 33-year-old man presented to the plastic surgery department for foreign body removal 1 month after the insertion of a CGM sensor (Dexcom G5, San Diego, CA, USA) in the left upper arm. The patient had used the CGM system for 5 years, and the insertion was done in the usual manner. After insertion, mild pain and redness at the insertion site continued for 3 days. At the patient's initial visit, no inflammatory signs were noted. The entire sensor wire was visible on simple radiography (Fig. 1A) and ultrasonography (Fig. 1B). In the operating room, the sensor wire was identified in the intermuscular septum and removed (Fig. 2A). No foreign body reaction or inflammatory signs were found around the foreign body. The foreign body

Copyright $(\odot 2021$ The Korean Society of Plastic and Reconstructive Surgeons

This is an Open Access article distributed under the terms of the Creative Commons Attribution Non-Commercial License (https://creativecommons.org/

licenses/by-nc/4.0/) which permits unrestricted non-commercial use, distribution, and reproduction in any medium, provided the original work is properly cited. I www.e-aps.org 

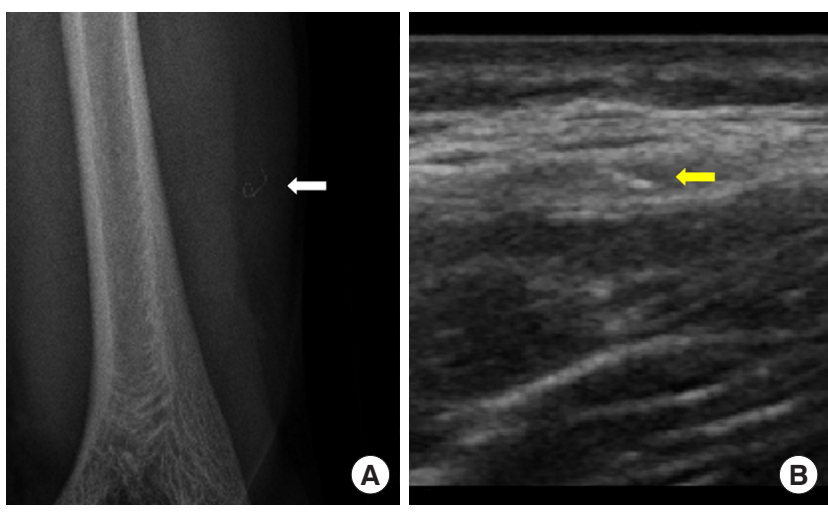

Fig. 1. Preoperative images. The sensor wire detached from the transmitter (white and yellow arrows) is shown on simple radiography $(\mathrm{A})$ and above the deep fascia on ultrasonography (B).

measured $2.5 \mathrm{~cm}$, leading us to assume that the entire sensor wire had detached from the transmitter, not fractured. The foreign body showed a darkish change in color after 1 month, compared with a new device (Fig. 2B). No remnant foreign body was found on follow-up simple radiography.

\section{DISCUSSION}

In recent years, various glucose-sensing mechanisms have been tested, including electrochemical, optical, and piezoelectric devices. The most widely used mechanism is the glucose oxidase electrochemical principle [4]. CGM devices consist of three parts (a sensor, transmitter, and receiver). This needle sensor is usually inserted in the subcutaneous tissue, in the abdomen, or on the arm. The sensor wires are made of electrodes containing glucose oxidase and are fixed on the sensor using chemicals imprinted on translucent plastic wafers. The sensor measures the electric current signal generated by the glucose oxidase reaction, which is proportional to the glucose level in the interstitial fluid [1].

The patient was an expert user of the CGM device, and the device had been inserted in the usual manner. Therefore, it is unlikely that the patient misused the device (e.g., by excessive pinching of the skin during insertion) as an explanation for the detached whole sensor wire. The process of using the device is simple, and the patient was skillful and used it in the routine manner; thus, we hypothesize that this case resulted from a device defect rather than misuse.

After 3 days of insertion into the subcutaneous layer, the sensor wire undergoes a fibrotic process that provides fibrotic encapsulation, which isolates it from the normal tissue [5]. We initially guessed that tracing the area of inflammation would make it easier to find the wire. However, encapsulation of sensor wire was

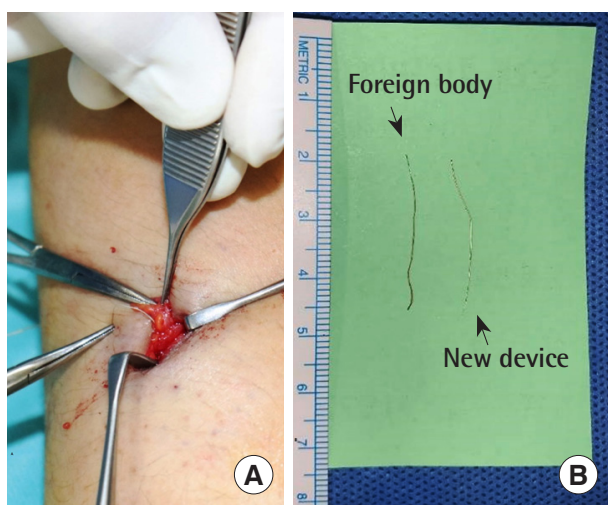

Fig. 2. Intraoperative images. (A) The sensor wire was identified in the intermuscular septum and removed. A foreign body reaction or inflammatory signs were not shown around the foreign body. (B) The foreign body measured $2.5 \mathrm{~cm}$, leading us to assume that the whole sensor wire had detached from the transmitter, not fractured. The foreign body showed a darkish color change over the course of 1 month, compared with a new device.

not identified and the surrounding tissue was not inflammatory, making it difficult to trace the wire. In this case, the sensor wire was identified on simple radiography and ultrasonography. However, the sensor wire was so thin that it was difficult to detect the sensor wire even using intraoperative radiography or ultrasonography. We continued to search for the localized area on radiography for a long time and eventually found the sensor wire.

It would have been easier to trace the sensor wire if a safety device had been attached to the wire. To the best of our knowledge, this is the first reported case of a CGM device complication resulting from a device defect. The development of CGM is in full swing and new device models are being introduced, but the possibility of this complication should be taken into account and patient education is needed.

\section{NOTES}

\section{Conflict of interest}

No potential conflict of interest relevant to this article was reported.

\section{Ethical approval}

The study was performed in accordance with the principles of the Declaration of Helsinki. Written informed consent was obtained.

\section{Patient consent}

The patient provided written informed consent for the publication and the use of his images. 


\section{Author contribution}

Conceptualization: HJ Choi. Project administration: HJ Choi. Visualization: KC Park. Writing - original draft: KC Park. Writing - review \& editing: KC Park. Approval of final manuscript: all authors.

\section{ORCID}

Kyong Chan Park https://orcid.org/0000-0002-0742-1233

Hwan Jun Choi https://orcid.org/0000-0002-0752-0389

\section{REFERENCES}

1. Cappon G, Acciaroli G, Vettoretti M, et al. Wearable continuous glucose monitoring sensors: a revolution in diabetes treatment. Electronics 2017;6:65.
2. Reddy N, Verma N, Dungan K. Monitoring technologies: continuous glucose monitoring, mobile technology, biomarkers of glycemic control. In: Feingold KR, Anawalt B, Boyce A, et al., editors. Endotext. South Dartmouth: MDText.com, Inc.; 2000. Available from: https://www.ncbi. nlm.nih.gov/sites/books/NBK279046/.

3. Wang Y, Vaddiraju S, Gu B, et al. Foreign body reaction to implantable biosensors: effects of tissue trauma and implant size. J Diabetes Sci Technol 2015;9:966-77.

4. Wang J. Electrochemical glucose biosensors. Chem Rev 2008;108:814-25.

5. McClatchey PM, McClain ES, Williams IM, et al. Fibrotic encapsulation is the dominant source of continuous glucose monitor delays. Diabetes 2019;68:1892-901. 